\title{
COMPARAÇÃO DAS CATEGORIAS ESPACIAIS POR MEIO DE SISTEMAS LINGÜISTICO E PICTÓRICO EM PRÉ-ESCOLARES DE DIFERENTES GRUPOS SÓCIO-ECONÔMICO-CULTURAIS *
}

Leonor Sclair Cabral **

Ana Maria de G. e Albuquerque Roncada $* *$ :

CABRAL, L. S. \& RONCADA, A. M. de G. e A. Comparacão das categorias espaciais por meio de sistemas lingüístico e pictórico em pré-escolares de diferentes grupos sócio-econômico-culturajs. Rev. Saúde públ., S. Paulo, 15(supl.):138-47, 1981.

RESUMO: Foi investigado um sistema discriminativo relacionado à psicolinguística a ser aplicado no Programa Centro de Educação e Alimentação dc Pré-Escolar (CEAPE). Optou.se por explorar a representação espacial por meio de sistemas lingüístico e pictórico. A amostra estudada foi constituída de 105 crianças de 3 a 6 anos e meio pertencentes a três grupos: do Programa CEAPE, de um grupo controle "Não-Ceapense" e de um outro de nível sócio-econômico alto da cidade de São Paulo. Estudou-se o poder discriminativo das perguntas nos três grupos de pré-escolares. Em relação às variáveis, nivel sócio-econômico-cultural e estimulação, houve diferenças significativas para lestes de compreensão lingüística em favor do grupo de alto nível sócio-econômico-cultural. As percentagens de categorias dominadas por $100 \%$ dos componentes dos três grupos foram, respectivamente: $41,7 \%$ (São Paulo), 30,6\% (CEAPE) e, 25,0\% (não CEAPE); 95\% dos componentes dos três grupos dcminaram, respectivamente, $50,0 \%$ (São Paulo), $47,2 \%$ (CEAPE) e $36,1 \%$ (não CEAPE) das categorias.

UNITERMOS: Pré-escolares, avaliação. Criança, desenvolvimento. CEAPE.

\section{INTRODUÇÃO}

Num programa (CEAPE) que se propõt, dentro das possibilidades existentes, diminuir a lacuna do curriculo escondido em população pré-escolar desde os dois anos, bem como suplementar a dieta alimentar, a contribuiçăo dos especialistas em psicolingüística, no presente experimento, consiste, numa primeira etapa em buscar uma medida discriminativa que possa ser facilmente aplicável como diagnóstico e, em decorrência, como fonte para exercicios adequados dentro do programa CEAPE.

Por isto, optou-se por explorar uma secção, a da representação do espaço através do sistema lingüístico e pictório.

Um modelo lingüístico foi utilizado para testar as categorias lingüísticas espaciais.

$\mathrm{Na}$ presente pesquisa foram observadas crianças pertencentes ao período pré-opera-

* Conrénio 10/77 - INAN/DN/FSP/USP.

** Da Pontifícia Universidade de Campinas - 13100 - Campinas, SP e da Escola Paulista de Medicina - Rua Botucatu, 720 - 04023 - São Paulo, SP - Brasil.

*** Da Pontificia Universidade Católica do Rio Grande do Sul - Ar. Ipiranga. 6.681 - Caixa Postal 1429 - 90000 - Porto Alegre, RS - Brasil 
CABRAL, L.S. \& RONCADA, A.M. de G. e A. Comparação das categorias espaciais por meio de sistemas lingüistico e pictórico em pré-escolares de diferentes grupos sócio-econômico-culturais. Rev. Saúde públ., S. Paulo, 15̃(supl.): 138-47, 1981

cional, uma vez que os sujeitos estavam na faixa etária dos três aos seis anos e seis meses de idade.

Em acréscimo à teoria de Piaget e Inhelder 20 (1948), levam-se em consideração, o fator histórico e, no mesmo contexto histórico, as diferenças encontradas entre os cule estão expostos a diferentes subsistemas (Pinxten 22, 1976). A presente hipótese de trabalho investiga a dependência da rapidez com que emergem as categorias espaciais ao fator estimulação e, igualmente, que, quanto mais complexas tais categorias e sua estruturação, tanto mais dependentes estarão de uma estimulação adequada.

Um outro ponto que merece consideração diz respeito às diferenças entre os diversos sistemas semiológicos, tais como as que há entre o lingüístico e o pictórico.

A hipótese (confirmada na presente pesquisa) é a de que existem limites e especificidades características a cada sistema, sendo a mais importante a de que no ato da fala, o emissor e o receptor estão presentes no ato da comunicação; as categorias espaciais estão referidas pelo sistema dêitico de pessoa que será analisado na secção seguinte.

A formalização das categorias de espaço e de localização por meio dos vários sistemas lingüísticos conforme Vernay 26 (1974), é um conjunto extraido das categorias universais potenciais da matriz cognitiva humana que é ativado e progressivamente aumenta de complexidade, através da acumulaçãn cultural *

Resumindo, toda vez que alguém procura localizar um objeto en relação a um interlocutor, poderá valer-se de duas maneiras (os chamados campos), o "Zeigfeld", definido pela expressão latina demonstratio ad oculos, na estrutura eu-aqui-agora e cuja significação é totalmente dependente da si- tuação. A segunda maneira é denominada de "Symbolfeld", isto é, de campo simbólico: neste sistema, as palavras se situam e se determinam em relação ao contexto lingüistico e situacional: elas não são utilizadas para apontar para as coisas e eventos, mas sim para representá-los.

No processo de aquisição de linguagem, as crianças desenvolvem este segundo sistema paralelamente, o que é uma condição para a depreensão da situação e relacionamento entre objetos e dos objetos e eventos que estão espacial e temporalmente ausentes. É, igualmente, uma condição para comunicar o ponto de vista do interlocutor e/ou de pessoas ausentes.

$O$ ponto de partida na presente pesquisa é, pois, a dêixis de localizaçāo e de pessoa.

A oposição básica na dêixis de localização ocorre entre o aqui e não aqui. O aqui é definido como o campo espacial compartilhado por ambos participantes do ato da fala, de modo contíguo.

Da intersecção desta oposição básica com a dêixis de pessoa, obtém-se uma outra oposição: aqui+eu / não aqui+eu. Postula-se que esta é a primeira oposição espacial na criança.

A seguir, a dêixis de localização e a dêixis de pessoa são enfeixadas com a de quantificação descontinua. Sendo assim, o tspaço passa a ser dividido em: coincidente, próximo e distante, em relação ao eu-aqui. A mesma divisão do espaço é atribuída às segunda e terceira pessoas do discurso, proporcionalmente ao desenvolvimento da descentração nas crianças.

Finalmente, uma outra noção, a de movimento, oposta à estática, completa o quadro, referencial.

\footnotetext{
* Para tornar esta seção de leitura mais compreensivel, não será utilizada a notação formal empregada por Vernay 26.
} 
CABRAL, L.S. \& RONCADA. A.M. de G. e A. Cumparação das categorias espaciais por melo de sistemas lingứístico e pictórico em pré-escolares de diferentes grupos sócio-econômico-culturais. Rev. Saúde públ., S. Paulo, 15(supl.): 138-47, 1981

MATERIAL E METODO

\section{Categorias mensuradas}

As seguintes categorias foram mensuradas:

eu/você/ele; aqui, ali, lá; este, esse, aquele; perto, longe; em cima de, embaixo de; em frente de, atrás de; à direita de, à esquerda de; em pé, deitado, sentado; para cima, para baixo; subir, descer; para frente, para trás; para a direita, para a esquerda; de, para, por, entre; dentro, fora.

As categorias topológicas foram estudadas apenas através do sistema pictórico e foram as seguintes: vizinhança, separação, relaçōes de ordem, envolvimento e descontinuidade.

A amostra estudada foi constituida de 105 crianças cujas idades variaram de 3 a 6,6 anos, divididas em 7 grupos etários, com 15 crianças por grupo etário, sendo $\mathbf{5}$ crianças para cada uma das amostras SAO PAULO, CEAPE e NÃO-CEAPE.

Os sujeitos pertenciam a dois diferentes grupos sócio-econômico-culturais: um alto (SÃO PAULO) e um baixo, subdividido, por seu turno em dois sub-grupos: um pertencente ao CEAPE (Centros de Educação e Alimentação do Pré-Escolar) (Gandra ${ }^{8}$, 1973) e que constituem um modelo alternativo de atendimento aos pré-escolares de 2 a 6 anos, a fim de thes oferecer atividades psico-pedagógicas na forma de recreação crientada e suplementação alimentar, e o outro subgrupo pertencendo também ao nível sócio-econômico-cultural baixo, sem estimulação NAO-CEAPE.

Duas pesquisadoras bem treinadas tomaram parte no experimento, uma delas dando instruções e a outra tomando notas cuidadosas.

$O$ teste somente foi aplicado após o estabelecimento de um bom relacionamento com as crianças e a certeza de que haviam entendido as instruções.
Os testes de compreensão lingüística foram baseados em comandos verbais que eliciassem respostas motoras.

O teste de compreensão no sistema pictórico utilizou estímulos verbais e pictóricos e uma resposta motora (apontar).

No teste de produção lingüistica, o principal objetivo foi testar o distanciamento (Brown ${ }^{1}$, 1973), de modo que as duas examinadoras estavam em salas diferentes. A primeira examinadora mostrava à criança a mesma situação do teste de recepção. Depois de mostrar cada uma das categorias, a criança deveria contar à examinadora que estava na outra sala o que tinha visto. Esta parte da pesquisa não será analisada aqui.

A produção pictórica foi levada a efeito em duas etapas: a primeira consistia em o examinador mostrar uma gravura e depois solicitar à criança que completasse o desenho.

A outra parte da produção pictórica consistia de um desenho livre onde as categorias topológicas foram examinadas. Um outro modelo (Olivier 17, 1974) também foi utilizado pará analisar estes desenhos que foram comentados em profundidade em Roncada ${ }^{2+}$ (1979).

\section{DISCUSSAO DOS RESULTADOS}

Em relação à primeira das indagações da presente pesquisa, a ordem de complexidade das categorias, os resultados mostraram que, no teste de compreensão lingüistica, $100 \%$ das crianças dominaram as seguintes categorias, independentemente do nivel sócio-econômico-cultural e da estimulação: ell-você-ele; em pé, deitado, em cima de, para; portanto, estas categorias não constituem medidas discriminativas nestas faixas etárias.

Há uma pequena diferença em favor das crianças estimuladas ("CEAPE" e "SAO PAULO"), em relação ao dominio $100 \%$ de categorias. 
CABRAL, L.S. \& RONCADA, A.M. de G. e A. Comparação das categorias espaciais por meio de sistemas linguístico e pictórico em pré-escolares de diferentes grupos sócio-econômico-culturais. Rev. Saúde públ., S. Paulo, 15(supl.): 138-47, 1981

As percentagens de categorias dominadas por $100 \%$ dos componentes dos três grupos foram, respectivamente: $41,7 \%$ (SAO PAULO), 30,6\% (CEAPE) e 25,0\% NAO-CEAPE).

Dos componentes dos três grupos dominaram $95 \%$ das categorias: $50,0 \%$ SAO PAULO, $47,2 \%$ CEAPE e $36,1 \%$ NÃO-CEAPE.

A noção de dentro no teste de compreensão lingüistica mostrou-se discriminativa no чue diz respeito à estimulação. Estes resultados confirmam os de Hunt e col12 (1975): no que diz respeito ao déficit para as informações atinentes à posição, resultantes de condições insatisfatórias durante o periodo pré-escolar. A ordem de domínio das categorias foi a mesma, principalmente para: dentro, em cima de, em frente de, entre, atrás de. Houve discrepância no conceito de em baixo de, uma vez que o grupo de nivel sócio-econômico-cultural baixo NAO-CEAPE saiu-se melhor que o CEAPE (este último com $90 \%$ de acertos).

A Tabela 1 mostra que a noção de por apresentou apenas $5 \%$ de respostas corretas por parte das crianças pertencentes ao nivel sócio-econômico-cultural baixo, independentemente de terem ou não treino especial. 0 grupo de nivel alto mostrou $45 \%$ de respostas corretas nesta categoria. Este conceito envolve os traços de origem, destino, retenção do âmbito espacial e movimento. Pesquisas subsequientes que observem a imagem e a representação lingüística do movimento deverão ser levadas a cabo para clarificar resultados conflitantes (Marmor ${ }^{16}$, 1975).

As outras categorias que se mostraram mais dificeis para as crianças foram, conforme era esperado, as noções de direita e esquerda. Maior número de acertos foi obtido nas camadas de mais alto nivel sócio-econômico (São Paulo). Hardy e col.10 (1974) chegaram às mesmas conclusões ao examinarem 50 crianças do Jardim de Infância.
E sobremaneira difícil para as crianças e mesmo para os adultos espelhar no refe$r \in$ te as noções retiradas do esquema corporal (Dubois ${ }^{6}$, 1975), já que, conforme tem sido bastante assinalado na literatura, o corpo humano apresenta simetria externa, embora funcionalmente ocorra dominância lateral esquerda para as funções de linguagem articulada, na maior parte dos individuos (Kershner 14, 1971; Goyvaerts 9, 1975). Apesar dos referentes utilizados no experimento serem orientados quanto à frente $e$ atrás (uma casa, um avião, um pássaro), estas categorias demonstraram ser muito difíceis. Harris e Strommen ${ }^{11}$ (1972) evidenciaram, contudo, que crianças entre quatro-oito anos apresentaram respostas concordantes sobre as partes fronteira e traseira dos objetos, tivessem eles pistas ou não, sendo as respostas respectivamente de $80 \%$ e $67 \%$. Mas Windmiller $2 \%$ (1976), ao estudar os pré-requisitos cognitivos para o entendimento do locativos espaciais, chegou a conclusões opostas.

Comentários adicionais devem ser feitos sobre o domínio das noções de aqui, ali $\mathrm{e}$ lá; este, esse, aquele; perto e longe. Pelo exame da Tabela 2 , os sujeitos não demonstraram um dominio mais precoce nestas categorias conforme era esperado. Deve-se, porém, ter em mente que estas eram as categorias nas quais as noções de egocentrismo/ponto de vista do interlocutor estavam sendo testadas. Existe uma clara relação entre idade e dificuldade em colocar-se no ponto de vista de outrem para localizar objetos no espaço, ainda que alguns pesquisadores estejam em desacordo com as posturas de Piaget sobre o relacionamento existente entre egocentrismo espacial e comunicativo (Rubin ${ }^{25}$, 1974). 
CABRAL, L.S. \& RONCADA, A.M. de G. e A. Comparaçäo das categorias espaciais por meio de sistemas lingüistico e pictórico em pré-escolare:3 de diferentes grupos sócio-econômico-culturais. Rev. Saúde públ., S. Paulo, 15(supl.): 138-47, 1981

\section{TA B E L A 1}

Categorias lingüisticas: Teste de compreensão aplicados a pré-escolares de diferentes grupos sócio-econômico-culturais, do Programa CEAPE - Municipio de Leme, SP.

\begin{tabular}{|c|c|c|c|}
\hline$\%$ & Grupo "NÃO-CEAPE" & Grupo "CEAPE" & Grupo "SÃO PAULO" \\
\hline \multicolumn{4}{|l|}{0} \\
\hline 5 & por & por & \\
\hline 10 & & à direita de & \\
\hline \multicolumn{4}{|l|}{15} \\
\hline 20 & $\begin{array}{l}\text { à direita de } \\
\text { para a direita }\end{array}$ & $\begin{array}{l}\text { à esquerda de } \\
\text { para a direita } \\
\text { para a esquerda }\end{array}$ & \\
\hline 25 & $\begin{array}{l}\text { à esquerda de } \\
\text { para a esquerda }\end{array}$ & & \\
\hline 30 & & esse - aquele & \\
\hline 35 & esse & & pora a esquerda \\
\hline 40 & longe (inter) & entre & à esquerda de \\
\hline 45 & aquele & & $\begin{array}{l}\text { para a direita } \\
\text { por }\end{array}$ \\
\hline 50 & este & ali - este & $\begin{array}{l}\text { este-esse-dquele } \\
\text { à direita de }\end{array}$ \\
\hline 55 & entre & & lá \\
\hline 60 & ali & & ali \\
\hline 65 & lá & perto-longe (inter) & aqui \\
\hline 70 & & lá & entre-longe (inter) \\
\hline 75 & aqui-perto (inter) & aqui & perto (exter) \\
\hline 80 & $\begin{array}{l}\text { em frente de } \\
\text { para trás }\end{array}$ & & para frente \\
\hline 85 & $\begin{array}{l}\text { longe (exter) } \\
\text { para frente }\end{array}$ & $\begin{array}{l}\text { longe (exter) } \\
\text { para trás }\end{array}$ & $\begin{array}{l}\text { atrás de } \\
\text { para trás }\end{array}$ \\
\hline 90 & $\begin{array}{l}\text { atrás de-descer } \\
\text { para baixo-subir } \\
\text { dentro }\end{array}$ & $\begin{array}{l}\text { atrás de } \\
\text { em baixo } \\
\text { para baixo }\end{array}$ & $\begin{array}{l}\text { perto (inter) } \\
\text { em frente de }\end{array}$ \\
\hline 95 & $\begin{array}{l}\text { perto (exter) } \\
\text { para cima } \\
\text { de - fora }\end{array}$ & $\begin{array}{l}\text { perto (exter) } \\
\text { em frente de } \\
\text { subir-descer } \\
\text { pra frunte-fora }\end{array}$ & $\begin{array}{l}\text { longe (exter) } \\
\text { sentado } \\
\text { de }\end{array}$ \\
\hline 100 & $\begin{array}{l}\text { eu-você-ele } \\
\text { em cima de-para } \\
\text { embaixo-sentado } \\
\text { em pé-deitado }\end{array}$ & $\begin{array}{l}\text { eu-rocê-ele } \\
\text { em cima de-para } \\
\text { para cima-sentado } \\
\text { em pé-deitado } \\
\text { de-dcntro }\end{array}$ & $\begin{array}{l}\text { eu-você-ele } \\
\text { em cima de-para } \\
\text { para cima-para baixo } \\
\text { em pé-deitado-dentro } \\
\text { embaixo-fora } \\
\text { subir-descer }\end{array}$ \\
\hline
\end{tabular}


CABRAL, L.S. \& RONCADA, A.M. de G. e A. Comparação das categorias espaciais por meio de sistemas lingǘístico e pictórico em pré-escolares de diferentes grupos sócio-econômico-culturais. Rev. Saúde puibl., S. Paulo, 15(supl.): 138-47, 1981.

\section{T A B E L A 2}

Proximidade versus não proximidade através de pro-locativos, a criança versus o interlocutor como centro ou referência e vice-versa, em pré-escolares segundo diferentes niveis sócio-econômico-culturais, do Programa CEAPE - Municípic de Leme, SP.

\begin{tabular}{llccc}
\hline $\begin{array}{c}\text { Proximidade } \\
\text { examinador/criança }\end{array}$ & Noções & $\begin{array}{c}\text { Grupo } \\
\text { "NAO CEAPE" }\end{array}$ & $\begin{array}{c}\text { Grupo } \\
\text { "CEAPE" }\end{array}$ & $\begin{array}{c}\text { Grupo } \\
\text { "SAO PAULO" }\end{array}$ \\
\hline Examinador perto & aqui & $80 \%$ & $80 \%$ & $85 \%$ \\
da criança & ali & $65 \%$ & $60 \%$ & $70 \%$ \\
& lá & $50 \%$ & $70 \%$ & $70 \%$ \\
Examinador longe & aqui & $40 \%$ & $65 \%$ & $50 \%$ \\
da criança & ali & $40 \%$ & $35 \%$ & $70 \%$ \\
& lá & $70 \%$ & $70 \%$ & $30 \%$ \\
\hline
\end{tabular}

Com relação às conclusões de De Villiers e De Villiers ${ }^{5}$ (1974), investigou-se no presente experimento o desempenho dos sujeitos em duas situaçóes contrastivas: uma na qual a examinadora estava ao lado da criança e outra quando estava frente a frente e longe dela. A posição vis-a-vis pode envolver espelhamento, um conceito cognitivo muito complexo que desempenha um papel na passagem de egocentrismo para o estádio não egocêntrico. Para explicar este fenômeno, considera-se a existência de uma reanálise feita pelas crianças em virtude da passagem do "Zeigfeld" para o "Symbolfeld", isto é, o que era utilizado como mera partícula de denominação agora passa a pertencer a um paradigma mais complexo, onde os traços de localização, pessoas do discurso e quantificação se interseccionam. Esta interpretação reforça a sugestão de Jackendoff ${ }^{13}$ (1976) sobre o desenvolvimento gradual nas crianças para domínios mais abstratos e abrangentes. Ao se analisar os dados de forma mais cuidadosa, pode-se verificar que todas as crianças mostraram um melhor desempenho quando eram o centro da referência (Tabela 2 ).

A ordem de aquisição dos conceitos espaciais que dizem respeito à verticalidade, perspectividade e lateralidade mostra que a noção de sobre foi a primeira a ser adquirida pelos três grupos. $O$ experimento não demonstra que as noções de polaridade sejam dominadas primeiro, já que há um desbordamento nos três grupos. Algumas pesquisas (veja-se Butterworth ${ }^{2}$, 1976) confirmam esta assimetria ontogeneticamente. Em cima parece ser a noção não marcada e mais simples. Embora Friedman e Seely ${ }^{7}$ (1976) ao discutir a hipótese de Clark ${ }^{3,4}$ (1973) de que as crianças entendam o membro positivo num par de antônimos, num teste de compreensão, desconfirmam assim a hipótese dos defensores da assimetria. Certamente a transposição do esquema corporal é compreendida mais facilmente no eixo vertical, começando com a noção da posição da cabeça.

A diferença crucial entre os dois sistemas semiológicos diz respeito à situação do discurso. $\mathrm{Na}$ comunicação oral, o emissor fala para um receptor presente sobre algo (presente ou ausente). Aí reside a origem da dêixis de lugar, pessoa (e tempo); a quantificação descontínua é também relacionada à dêixis.

Nas gravuras, a quantificação descontínua apenas pode ser dividida em proximidade e distância (a coincidência é impossível) 
CABRAL, L.S. \& RONCADA, A.M. de G. e A. Comparação das categorias espaciais por meio de sistemas linguístico e pictórico em pré-escolares de diferentes grupos sócio-econômico-culturais. Rev. Saúde publ., S. Paulo, 15(supl.): 138-47, 1981

entre objetos e eventos representados no espaço pictórico, através dos traços de projeção e perspectividade.

A noçào de aqui foi dominada com algum desbordamento. $\mathrm{O}$ grupo mais haixo sem tتieinamento especial começou a dominá-la no grupo de 4,6 a 5 anos con um pequeno decréscimo no grupo de 6 a 6.6 anos. 0 grupo CEAPE começou a dominar a categoria no grupo de 3,6 a 4 anos, com pequeno decréscimo nos grupos de 4 a 4,6 anos e de 5,6 a 6 anos. $O$ grupo pertencente ao nivel sócio-econômico-cultural mais alto mostrou o pior desempenho: apenas os grupos de 5 a 5,6 e 5,6 a 6 anos mostraram o domínio completo desta categoria.

Ao se comparar, contudo, o domínio da noção de aqui com a de ali e lá, conclui-se que o paradigma não está completamente organizado nas crianças que pertencem ao grupo sem treinamento intensivo, enquanto que alguns grupos do CEAPE mostraram a distinção entre aqui e ali e alguns dos mais altos mostraram desempenho mais homogêneo.

A noção de aqui mostra resultados inconsistentes e foi testada deliberadamente para confirmar a hipótese de ausência da dêixis de pessoa no sistema pictórico (excluídas as estórias em quadrinhos).

Outras categorias dominadas primeiramente pelas crianças no teste de recepção foram: em baixo de, em cima de, de pé, deitado, sentado, dentro, seguidas por fora. Existem categorias que correspondem a referentes de uma natureza mais concreta: são mais fáceis de serem percebidos. Estas categorias também foram dominadas mais cedo no teste lingüístico.

A noção de de (procedência), uma das primeiras a ser dominada no teste lingüstico, mostrou-se difícil no teste pictórico. A explicação para este caso específico é a dificuldade para representar pictoricamente conceitos de atravessar, que contêm origem, movimento e alvo.

A análise dos desenhos a fim de testar as categorias topológicas confirma a hipó- tese de que vizinhança é a primeira categoria a ser adquirida pelas crianças. A noção de separação mostrou algum desbordamento: as crianças pertencentes ao grupo CEAPE apenas dominaram completamente esta categoria aos 5,6 a 6 anos, diferentemente dos dois outros grupos que dominaram antes ( 4 a 4,6 anos). As categorias de ordem e entre, conforme o esperado, estão muito relacionadas, mostrando um desenvolvimento similar.

A noção de envolvimento foi a última a ser dominada pelos três grupos. Os resultados estão concordes com a hipótese de Piaget e col.21 (1964), sobre a ordem de emergência das categorias topológicas, exceto quanto à de envolvimento; também estâo concordes com a asserção daqueles autores de que os sistemas de referência bidimensionais se desenvolvem durante $o$ periodo do pensamento operacional concreto (veja-se igualmente Pufall e Shaw 23, 1973).

Comparando os três grupos em seu desempenho como um todo, pode-se perceber claramente que o nível sócio-econômico-cultural mais baixo, sem treinamento intensivo (NAO-CEAPE), mostrou desempenho mais pobre se comparado com os outros dois grupos. Comparando os resultados com os obtidos dos testes lingüísticos, pode-se concluir que a estimulação desempenhou um papel mais importante nas capacidades pictóricas que lingüisticas, confirmando a hipótese inicial. Outros autores como Omari is (1975) assinalam a influência dos fatores ambientais sobre a aquisição dos conceitos espaciais entre escolares na Tanzânia, e Page ${ }^{19}$ (1973), que conduziu um experimento entre jovens zulus, demonstrou as dificuldades que as populaçōes rurais apresentaram ao passar das categorias topológicas para as euclidianas. Kershner 15 (1972) conclui que as crianças américo-hispânicas apresentam comportamento analítico-espacial, enquanto as anglo-saxônias mostraram um comportamento verbal global, cuando testadas sobre suas capacidades para reproduzir direção e orientação. 
CABRAL, L.S. \& RONCADA, A.M. de G. e A. Comparacão das categorias espaciaịs por meio de sistemas lingüistico e pictórico em pré-escolares de diferentes grupos sócio-econômico-culturais. Rev. Saüde públ., S. Paulo, 15(supl.): 138-47, 1981

\section{CONCLUSOES}

Para os propósitos do presente experimento, as seguintes conclusões são relevantes:

a) Nos testes de compreensão lingüística, nas faixas etárias dos 3 aos 6,6 anos, não são discriminativas as categorias espaciais relativas a por, $\dot{a}$ esquerda de, à direita de, para a esquerda e para a direita, no nivel sócio-econômico-cultural mais baixo, independentemente da estimulação.

b) As categorias eu-você-ele, em pé, deitado, em cima de, para não são discriminativas para todos os grupos examinados, por serem dominadas independentemente das faixas etárias examinadas, do nível sócio-econômico-cultural e da estimulação.

c) Os pronomes e advérbios de lugar relacionados às pessoas do discurso, quando se mede a criança já se coloca na posição do interlocutor, são discriminativos. d) A ordem de aquisição das noções topológicas sugeridas por Piaget foi confirmada no experimento, exceto a noção de envolvimento, que se mostrou a mais difícil.

e) Em relação às variáveis nivel sócio-econômico-cultural e estimulação, as diferenças mais significativas nos testes de compreensão lingüistica foram as registradas entre os grupos NAOCEAPE/CEAPE versus SAO PAULO, já que as categorias menos dominadas pelos primeiros grupos apresentaram $5 \%$ de acertos (a noção por) contra $35 \%$ de acertos no último grupo na noção menos dominada: para a esquerda. Ao se compararem os dois grupos de nível sócio-econômico mais baixo entre si, o NAO-CEAPE mostrou o número mais reduzido de categorias dominadas cem por cento: $25,0 \%$ contra $30,6 \%$ do CEAPE.

f) A estimulação sistemática e intensiva desempenhou um papel mais importante no desenvolvimento das capacidades pictóricas do que nas lingüisticas.

CABRAL, L. S. \& RONCADA, A. M. de G. e A. IComparison of spacial categories in preschool childreu by means of linguistic and pictorial methods in three different socio-econimic-cultural grcups] Rer. Saúde públ., S. Paulo, 15(suppl.): $138-47,1981$.

ABSTRACT: A research into a system, related to psycholinguistics, which is to be applied to a CFAPE program for preschool-age children is described. The choice fell upon spacial representation through linguistic and pictorial systems. A cross-section of ages was studied (from 3 to 6). Sample children came from 3 groups: those from a CEAPE program, a control group of children not covered by CEAPE and a high level socio-economic group from the city of $\mathbf{S}$. Paulo, Brazil. The differentiating power of the items was studied in the 3 groups. With regard to socio-economic-cultural level and stimulation, significant dufierences in linguistic understanding to the advantage of the high level socio-economic-cultural group were observed Percentages of categories in which all subjects, answered correcty were respectively: $41.7 \%$ (S. Paulo), 30.6\% (CEAPE) and 25.0\% (non-CEAPE). Ninety-five percent of the members of the 3 groups gave correct answers to $50.0 \%$ (S. Paulo), $47.2 \%$ (CEAPE) and $36.1 \%$ (non-CEAPE) of the items, respectively.

UNITERMS: Preschool, child, evaluation. Child development. CEAPE. 
CABRAL, L.S. \& RONCADA, A.M. de G. e A. Comparação das categorias espaciais por meio de sistemas lingüístico e pictórico em pré-escolares de diferentes grupos sócio-econômico-culturais. Rev. Saúde públ., S. Paulo, 15(supl.): 138-47, 1981

\section{REFERENCIAS EJBLIOGRÁFICAS}

1. BRoWN, R. A first language, the early stages. Cambridge, Mass., Harvard Untversity Press, 1973.

2. BUTtERWORTH, G. Assymetrical search errors in infancy. Child Develop. $47: 864-7, \quad 1976$

3. CLARK. E.V. What's in a word? On the child's acquisition of semantics in his firs language. In: More, T.E.. ed. Cognitive development and the acquisition of language. New York, Academic Press, 1973. p. 65-110.

4. CLARK, H.H. Space, time, semantics and the child. In: More, T.E., ed. Cognitive development and the acquisition of language. New York, Academic Press, 1973. p. 27-63.

5. DE VILLIERS, P.A. \& DE VILLIERS. J.G On this, that and the other: nonegocentrism in very young children. $J$. exper. Child. Psychol. 18:438-47, 1974.

6. DUBOIS, G. Orientation spatiale et temporalle: utilization d'epreuves Borel-Maisonny questionnaires espace et temps. Rev. Laring. Otol. Rhin., 96:375-472. 1975.

7. FRIEDMAN, W.S. \& SEELY, P.B. The child's acquisition of spatial and temporal word meanings. Child Develop., 47:1103-8, 1967.

8. GANDRA, Y.R. Asistencia alimentaria por medio de centros de educación $y$ alimentación del pre-escolar. Bol. Ofic. sanit. panamer., $\boldsymbol{7 4 : 3 0 2 - 1 4 ,} 1973$.

9. GOYVAERTS, M. Recent advances in language acquisition. Commun. \& Cogn., $8(1): 45-87, \quad 1975$

10. HARDY, M. et al. Development of auditory and visual language concepts and relationship to instructional strategies in kindergarten. Elem. Engl,, 51:525-32. 1974.

11. HARRIS, L.J. \& STROMMEN, E.A. The role of front-back features in children's front, back and beside placements of objects, Merril-Palmer Quart., 18:259-71. 1972.
12. HUNT, J.M. et al. Social class and preschool language skill. III - Semantic mastery of position information. Gen. Psychol. Monogr., 91:317-37, 1975

13. JAKENDOFF, R. Toward a cognitive riable semantics; Georgetown Round Table on Language and Linquistics. Washington D.C., Georgetown University Press, 1975

14. KERSHNER, J.R. Children acquisition of risuo-spatial dimensionality: a conservation study. Devclop. Psychol, 5:454-62, 1971

15. KERSHNER, J.R. Ethnic group differences in children's ability to produce direction and orientation. J. soc. Psychol., 88:3-13, 1972.

16. MARMOR. G.S. Development of kinetic images: when does the child first represent movement in mental images. Cogn. Psychol., \%:548-59, 1975.

17. OLIVIER, F, Le dessin enfantin est-il une ecriture? Paris, PUF, 1974. p. 183-216.

18. OMARI, I.M. Development order of spatial concepts among schoolchildren in Tanzania. J. Cross-Cult. Psychol., 6:444-56, 1975

19. PAGE, H.W. Concepts of length and distance in a study of Zulu youngs. $J$ soc, Psychol, 90:9-16, 1973.

20. PIAGET, J. \& INHELDER, N. La représentation de l'espace chez l'enfant. Paris. PUF, 1948.

21. PIAGET, J. et al. L'epistémologie de l'espace. Paris, PUF: 1964.

22. PINXTEN, R. Epistemic universals. A contribution to cognitive anthropology: In: Pinxten, R. ed. Universalism versus relativism in language and thought. The Hague, Mouton, 1976. p. 117-75.

23. PUFALL, P.B. \& SHAW, R.E. Analysis of the development of children's spatial reference systems. Cogn.Psychol., 5:151$-75,1973$. 
CABRAL, L.S. \& RONCADA, A.M. de G. e A. Comparação das categorias espaciais por meio de sistemas lingüistico e pictórico em pré-escolares de diferentes grupos sócio-econômico-culturais. Rev. Saúde públ., S. Paulo, 15(supl.): 138-47, 1981

24. RONCADA, A.M.G.A. Aquisição dos sistemas semiológicos lingüístico e pictório da espacialidade: possibilidade de transcodificação. Porto Alegre, 1979. [Dissertação de Mestrado - Pontificia Universidade Católica do Rio Grande do Sul]

25. RUBIN, K.H. The relationship between spatial and communicative egocentrism in children and young and old aduits. $J$. genet. Psychol., 125:295-301, 1974.
26. VERNAY, H. Essay sur l'organization de l'espace par divers systèmes linguistiques. Munchen. Wilhelm Eink Verlag. 1974.

27. WINDMILLER, M. A child's conception of space as a prerequisite to his understanding of spatial locatives. Genet. Psychol. Monogr., 94:227-48, 1976.

Recebido para publicação em 10/0\%/1981 Aprovado para publicação em 17/11/1981 